\title{
Introduction for inflammation and cancer
}

\author{
Takuji Tanaka
}

Received: 6 December 2012 / Accepted: 14 December 2012 / Published online: 9 January 2013

(C) Springer-Verlag Berlin Heidelberg 2012

How inflammation protects or injures tissues of the human body is quite an important issue, particularly in cancer development. Inflammation also affects the immune system or immune surveillance. We now know the importance of inflammation and the innate/adaptive immune system in tumor development, promotion, progression, and metastasis.

Inflammation has two faces in human tissues. The acute inflammatory response is the first system of alarm signals that are directed toward elimination of microbial invaders and wounded necrotic debris. Chronic inflammation has emerged as the pathophysiological basis of many chronic cardiovascular and neurodegenerative diseases that were not initially thought to be linked to an inflammatory response. It has now become apparent that inflammation is an important component of cancer development. This inflammation is now regarded as a "secret killer" of chronic diseases, such as cancer.

The concept of a relationship between inflammation and carcinogenesis is not new. Based on the presence of leukocytes within cancer tissue, the founder of cellular pathology, Dr. Rudolph Virchow, speculated that there was an association between inflammation and cancer development in 1863. In line with his notion, epidemiological data indicate that inflammation serves as a potential risk factor for the development of cancer in different tissues. It is generally

This article is a contribution to the special issue on "Inflammation and Cancer-Guest Editor: Takuji Tanaka."

\footnotetext{
T. Tanaka $(\bowtie)$

Tohkai Cytopathology Institute: Cancer Research and Prevention (TCI-CaRP), Gifu, Japan

e-mail: takutt@toukaisaibou.co.jp

T. Tanaka

Department of Pathology, Asahi University,

Gifu, Japan

T. Tanaka

Department of Tumor Pathology, Gifu University

Graduate School of Medicine, Gifu, Japan
}

accepted that up to $25 \%$ of human malignancies are related to chronic inflammation with or without (viral, bacterial, or parasitic) infections. Evidence shows that inflammatory stimuli caused by inflammation increase the risk of cancer, promote tumor progression, and support metastatic spread. Thus, inflammatory cells and cytokines act as tumor promoters that affect cell survival, proliferation, invasion, angiogenesis, and resistance of chemotherapy.

The immune system always acts to prevent preneoplastic cells progressing to cancer. On the other hand, cancer cells possess immunosuppressive properties such that they escape immune surveillance. In addition, we know that environmental factors decrease the immune function, resulting in cancer development. These observations together indicate that analysis of the immune function could contribute to the early detection, prevention, and treatment of cancer.

Based on the types of inflammation associated with carcinogenesis, the tumor microenvironment can include cancer cells, innate immune cells (macrophages, neutrophils, mast cells, dendritic cells, natural killer cells, and myeloidderived suppressor cells), adaptive immune cells ( $\mathrm{T}$ and $\mathrm{B}$ lymphocytes), and stromal cells (fibroblasts, vessel endothelial cells, and other mesenchymal cells). To maintain the growth of cancer, these cells communicate with each other directly or indirectly by producing cytokines and chemokines in autocrine and/or paracrine manners.

This special issue entitled "Inflammation and Cancer" is mainly focused on this topic but also touches on the prevention of inflammation-related cancers. Several worldclass experts from diverse fields and institutions have written review articles for this introductory special issue of the journal. Each of the reviews is presented as an introduction by experts who are involved in cutting-edge research in their area of expertise. Dr. Sven Brandau et al. describe the protumor and anti-tumor functions of neutrophil granulocytes in tumorigenesis. They propose a model where homeostatic chronic recruitment and activation of neutrophils result in 
mainly pro-tumor activity. In contrast, therapeutic interventions that enhance direct effector functions and indirect regulatory functions of neutophils result in potent antitumor activity. They postulate that conversion of protumor activity of neutrophils into anti-tumor activity may offer a novel biological therapy of cancer. Drs. Masako Nakanishi and Daniel W. Rosenberg summarize the effects of a bioactive lipid, prostaglandin E2 $\left(\mathrm{PGE}_{2}\right)$, in gastrointestinal carcinogenesis and discussed the complex and interconnected pathways that link $\mathrm{PGE}_{2}$ signaling with inflammation and cancer. Dr. Sergei Grivennikov reviews pathobiology of colitis-associated colorectal cancer in the large bowel. He introduced microbes as new players capable of triggering inflammation and possibly promoting carcinogenesis in the inflamed colon. Drs. Shmizu et al. postulate an association between obesity and hepatocellular carcinoma (HCC). They suggest multiple mechanisms, by which obesity influences HCC development, focusing on the emergence of insulin resistance and the subsequent activation of the inflammatory cascade. They also propose that nutritional or pharmaceutical approaches for preventing obesity-related liver carcinogenesis can be achieved by targeting pathophysiological conditions caused by obesity. Drs. Hiroko Oshima and Masanobu Oshima describe their own data regarding the role of $\mathrm{PGE}_{2}$-induced inflammatory responses in gastric cancer development using their genetically altered mice, called as Gan mice. They demonstrate novel molecular pathways that are activated or suppressed in $\mathrm{PGE}_{2}$-associated inflammatory microenvironment and thereby contribute to gastric cancer development. Dr. Mami Takahashi et al. describe pancreatic carcinogenesis, focusing on the roles of chronic inflammation and inflammatory factors in pancreatic carcinogenesis and the possible prevention of pancreatic ductal cancer using certain anti-inflammatory agents. They introduce interesting findings that a high-fat diet enhances hamster pancreatic carcinogenesis, along with aggravation of hyperlipidemia and severe fat infiltration (also observed in obese and diabetic patients) and increased expression of adipokines and inflammatory factors in the pancreas. Dr. Tsukamoto et al. report the role of Helicobacter pylori (H. pylori) infection in gastric carcinogenesis by using a novel experimental model, in which $H$. pylori infection induces intestinal metaplasia and intestinalization of stomach cancers independently. Finally, they mention that oxygen radical scavengers, anti-inflammatory chemicals, and the eradication of $H$. pylori are effective to prevent $H$. pylori-associated gastric carcinogenesis. Drs. Takuji Tanaka and Hideki Ishikawa present novel data on the role of mast cells in a mouse model of inflammation-associated colorectal carcinogenesis. They show that mice lacking mast cells are less susceptible to colitis-related colorectal tumorigenesis. Dr. Lee et al. review an association between inflammation and cancer development. They point out that inflammation (chronic inflammation) is a predisposing factor to carcinogenesis. They also emphasize that resolution of chronic inflammation is obtained by coordinated processes regulated by distinct anti-inflammatory and pro-resolving endogenous lipid mediators, including resolvins and lipoxins.

The aim of the guest editor is to provide a springboard to the ideas of new investigators currently devoted to cuttingedge research in these areas of inflammation and cancer. It exposes the reader to the exciting and fascinating cellular and molecular events that are involved in inflammationassociated carcinogenesis in a variety of tissues. I trust that the reader will share our enthusiasm and continued excitement for studying the cellular and molecular events in inflammation-associated carcinogenesis. Finally, we do hope that strategies to prevent or treat cancers will be developed in the near future based on insights into inflammatory pathways. 